\title{
Falsos cognados. Falsos problemas. Un aspecto de la enseñanza del español en Brasil*
}

\author{
Philippe Humblé \\ Universidade Federal de Santa Catarina
}

Es con este título un tanto provocativo que me gustaría llamar la atención sobre un problema que, en la enseñanza del español en Brasil, se ha recalcado mucho, como si fuera el mayor problema de todos: los falsos cognados ${ }^{1}$. El problema parece incluso tan crucial que se le han dedicado varios diccionarios y numerosos sitios en Internet.

¿Qué es lo que se suele indicar con este término? Es lo que a veces se usa graciosamente en anuncios para cursos de lenguas: confusiones entre palabras como "exquisito/esquisito", "embarazada/embaraçada" y otros. Mi hipótesis es que, en realidad, la reducción de los "falsos amigos", o "falsos cognados" a este tipo de ocurrencia esconde el verdadero problema, mucho más sutil, que es que el portugués y el español son lenguas diferentes y que toda desconfianza es poca. El portugués y el español se parecen mucho, pero a veces de una manera inesperada.

Hay en Internet un buen número de $\operatorname{sitios}^{2}$ que se dedican a avisar al alumno brasileño de los obstáculos que tendrá que salvar al estudiar el castellano. Un sitio como Kactuz enumera 116 palabras fácilmente confundibles. Otros sitios dan listas parecidas, generalmente más cortas. Se puede observar que es un número de palabras que cualquier estudiante brasileño podría aprender de memoria en dos tardes.

Hay también los diccionarios de falsos amigos, o de falsos cognados, de los que se editaron algunos en Brasil. Estos diccionarios son más serios que los sitios de Internet y listan un número mucho más extenso de casos. Estas listas, sin embargo, también son criticables, porque parten del presupuesto de que lo que se tiende a confundir son únicamente palabras que tienen una forma igual, pero de significado

\footnotetext{
* Agradezco a la CAPES la beca postdoctoral (2003-2004) que me ha posibilitado llevar a cabo la investigación necesaria para escribir este artículo.

1 Para una discusión sobre el término remito al lector al excelente artículo al respecto de Sabino (2002). La autora hace una distinción entre "falsos cognados", que se parecen pero no tienen ningún sentido en común, con los “cognados engañosos" que provienen de un mismo étimo, pero que sufrieron cambios de sentido a lo largo de los siglos.
}

2 Por ejemplo: http://www.espanholpro.vpg.com.br/falsos_cognatos.htm 
distinto. No solamente este no es, a mi modo de ver, el problema principal, sino que es una faceta del problema que no implica grandes peligros comunicativos. Efectivamente, los falsos cognados españoles y portugueses pocas veces ocupan lugares sintácticos idénticos, por lo que la posibilidad de confusión es mínima. "Borracha" (esp.) significa algo más bien diferente de "borracha" ('caucho' en portugués), pero como la distribución de estas dos palabras es diferente, parece improbable que dé algún día origen a malentendidos ${ }^{3}$. No es mi intención, en este artículo, tratar de demostrar que los falsos cognados no son un problema en la enseñanza del español a lusohablantes, sino todo lo contrario. Es el problema principal, pero pocas veces se identificó de una manera adecuada. El asunto es más complejo porque es, en gran parte, insospechado. Lo que vuelve el aprendizaje del español verdaderamente arduo por parte de los estudiantes brasileños son, en realidad, las palabras que suenan lo mismo, se escriben igual, significan lo mismo, pero se usan de una manera distinta. Distingo a estos efectos cuatro tipos de "falsos amigos".

- El primer tipo es el tradicional: dos palabras son casi idénticas, se escriben casi igual, se pronuncian casi igual, pero sus sentidos son diferentes. El ejemplo clásico es el de exquisito/esquisito.

- El segundo tipo es el de palabras que también son casi idénticas, significan lo mismo, pero que se usan en registros diferentes. Es el caso de, por ejemplo, aclarar y esclarecer.

- El tercer tipo es el de palabras que también son idénticas y significan grosso modo lo mismo, pero que tienen varias acepciones de las cuales una o algunas son diferentes. Es el caso de lástima.

- El cuarto tipo es el de palabras que forman parejas en cada una de las lenguas, con acepciones aparentemente idénticas, pero que funcionan en realidad con restricciones gramaticales o léxicas diferentes. Es el caso de $h a$ blar/decir, falar/dizer.

Para investigar estas diferentes posibilidades usé, por una parte, un corpus personal de 13 millones de palabras en español. Este corpus se compone mayoritariamente de textos periodísticos latinoamericanos y españoles. Por otra parte usé un corpus de portugués, de un número comparable de palabras, que se compone de textos de la Folha de São Paulo ${ }^{4}$ Usé también un pequeño corpus de lengua habla-

3 Podría objetarse secundariamente que es condición necesaria para el uso de estos diccionarios que el usuario sepa, o por lo menos sospeche, que la palabra que busca en el diccionario es efectivamente un falso cognado.

4 Dispongo, en realidad, de un corpus de casi 100 millones de palabras, también de la Folha de Sao Paulo, que usé para sacar más ejemplos de uso. Empleé, sin embargo, como corpus piloto uno de tamaño igual al que tenía en español, para que los números fueran comparables. 
da de portugués brasileño, de 3 millones y medio de palabras, proveniente del Proyecto Varsul 5 .

Huelga decir que eché mano igualmente, además de estos córpora, de la máquina de busca Google para verificar los resultados obtenidos a partir de los córpora.

\section{FALSOS AMIGOS EN EL REGISTRO: ACLARAR/ESCLARECER}

Un primer ejemplo de falsos cognados insospechados son los verbos aclarar y esclarecer. Por lo que se lee en los diccionarios que consulté, se les podría considerar sinónimos en castellano y en portugués, y ambas palabras serían, por lo tanto, traducciones satisfactorias una de otra: aclarar de aclarar, y esclarecer de esclarecer. Sin embargo, la realidad del uso no apunta en esta dirección. Al echar un vistazo al corpus, notamos que aclarar, en español, ocurre en la forma del infinitivo una vez a cada 63004 palabras. En portugués, sin embargo, aclarar ocurre una vez a cada 7647853 palabras, o sea, 125 veces menos. En el caso de esclarecer, se da el caso contrario. Esclarecer ocurre en español una vez a cada 235263 palabras, y una vez a cada 59632 palabras en portugués. O sea, esclarecer es 3.7 veces más frecuente en portugués que en español.

¿Qué se puede deducir de estas cifras? Primero, que esclarecer y aclarar no son simplemente intercambiables en las dos lenguas, si su frecuencia es diferente. Aclarar no es aclarar, y esclarecer no es esclarecer. Segundo, no por eso se puede decir que aclarar en una lengua equivale a esclarecer en la otra, y viceversa. Hay aparentemente otros verbos que ocupan parte del campo semántico, tal como clarificar, explicar, definir, demostrar, ilustrar.

Esclarecer, en español, es lo que se hace más que nada con asesinatos. Aclarar es lo que se hace con dudas e ideas. En portugués, por otro lado, aclarar se usa poquísimo, y no encontré ningún sustantivo con el que el verbo se usaría específicamente. Esclarecer, por el contrario, se usa muchísimo, más que nada con palabras como dúvidas, pontos y fatos, en ese orden de frecuencia. Encontré, sin embargo, casos de esclarecer con asesinatos también, como en español. En portugués, finalmente, se aclaran más que nada coisas y questões. En el caso de la palabra dudas, el verbo que se usa más en español es aclarar, y en portugués esclarecer. Sin embargo, en el caso de la palabra asesinato, en castellano, y assassinato, en portugués, el verbo puede ser esclarecer en ambas lenguas.

O sea, aclarar y esclarecer son verdaderos falsos cognados, pero esto no significa que esclarecer pueda traducirse por aclarar, y viceversa. El cuadro es, por lo tanto, complejo. Si alguien dice en castellano que "todas las dudas fueron esclareci-

5 El Proyecto Varsul estudia la variación lingüística que ocurre en los estados brasileños de Paraná, Santa Catarina y Rio Grande do Sul. 
das", será entendido correctamente, pero no se habrá expresado de una manera considerada natural en el contexto. Ahora, tal como era de esperarse en una comunidad lingüística de tantos países, hay reductos en que sí se puede "esclarecer una duda"6. Google nos da algunos ejemplos. Hay dos cubanos, tres argentinos, un español, un chileno, y un venezolano. O sea: se dice, pero no mucho.

Resumiendo, ¿en qué sentido aclarar y esclarecer son "falsos amigos”? En el sentido en que significan lo mismo, pero tienen un matiz de significado diferente en cada lengua. La frecuencia suele acompañar al registro. Palabras de registro más alto suelen ser menos frecuentes que palabras comunes, aunque esto dependa de la composición del corpus. Además, hay palabras extremadamente vulgares que no son, felizmente, muy comunes. Generalmente, sin embargo, una palabra poco frecuente es una palabra rebuscada, más elegante, más refinada. Puede ser que "esclarecer una duda" sea perfectamente aceptable en castellano, pero puede no ser una traducción exacta en lo que a registro se refiere. Hay centenares, incluso miles, de "amigos falsos" de este tipo en portugués y español, que no hacen que nos entendamos mal, sino que hacen que no nos comuniquemos de una manera adecuada.

\section{ESTE, ESE, AQUEL}

Ninguno de los diccionarios de falsos cognados que yo conozca menciona el problema de los pronombres y adjetivos demostrativos. Sorprende, sin embargo, el uso que los alumnos brasileños muchas veces hacen de estas partículas, un uso rápidamente incorporado por los hispanohablantes que se vienen a vivir en Brasil. Así no es infrecuente escuchar a alguien hablando de "ese teléfono", cuando el aparato está a su lado. Según las gramáticas tradicionales el uso de esos pronombres es, sin embargo, idéntico en ambas lenguas. Este corresponde a lo que está cerca del locutor, ese a lo que está cerca de la persona con la que estamos hablando, y aquel a lo que está alejado de los dos (Cunha y Cintra 1985: 3219; Alcina y Blecua 1975: 598). Pero la realidad parece ser un poco diferente. En portugués los pronombres este y esse se volvieron casi intercambiables, y esto no es el caso en castellano. ¿Qué dicen los números? En el cuadro siguiente registré las frecuencias de los pronombres demostrativos españoles y portugueses como aparecen en los córpora que usé.

6 Esto, además, ocurre frecuentemente. Uno corrige a un estudiante que al día siguiente le viene con ejemplos, sacados del Google, donde se dice exactamente lo que el profesor le acaba de corregir. Al final, en el caso de una lengua de una extensión tan grande, siempre se dice de todo en algún lugar. El caso es que de esa manera se puede justificar casi todo el portuñol, porque siempre "se dice en algún sitio". 


\begin{tabular}{|c|c|c|}
\hline & Español escrito & Portugués escrito \\
\hline Este & $1 / 548$ & $1 / 4963$ \\
\hline Estos / Estes & $1 / 1895$ & $1 / 27690$ \\
\hline Esta & $1 / 713$ & $1 / 6862$ \\
\hline Estas & $1 / 2275$ & $1 / 42224$ \\
\hline Promedio & $1 / 1357$ & $1 / 20434$ \\
\hline Relación Esp/Port & 15 & \\
\hline Ese/esse & $1 / 1365$ & $1 / 3164$ \\
\hline Esos/esses & $1 / 4270$ & $1 / 6631$ \\
\hline Esa/essa & $1 / 1526$ & $1 / 3395$ \\
\hline Esas/essas & $1 / 5583$ & $1 / 7178$ \\
\hline Promedio & $1 / 3186$ & $1 / 5092$ \\
\hline Relación Esp/Port & 1.59 & \\
\hline Aquel/aquele & $1 / 10679$ & $1 / 14177$ \\
\hline Aquellos/aqueles & $1 / 10276$ & $1 / 17960$ \\
\hline Aquella/aquela & $1 / 14741$ & $1 / 19203$ \\
\hline Aquellas/aquelas & $1 / 23834$ & $1 / 53110$ \\
\hline Promedio & $1 / 14882.5$ & $1 / 26112$ \\
\hline Relación Esp/Port & 1.75 & \\
\hline
\end{tabular}

FIG. 1. Comparación de demostrativos en los córpora

Con estos números no pretendo dar un panorama exacto del uso de los demostrativos en portugués y español, solo quiero mostrar algunas tendencias. Lo primero que se puede observar es que aparentemente hay una tendencia mucho más pronunciada en español que en portugués de usar demostrativos, en general. Aun en el caso de aquel, donde las cifras son más comparables, la frecuencia en español es casi el doble de la frecuencia en portugués. Esto, en sí, ya es una indicación de que se trata de falsos amigos, ya que aparentemente estas partículas se tienen que usar más en una lengua que en otra. No tienen, por lo tanto, el mismo valor semántico. Detengámonos brevemente en el caso de este/este y ese/esse.

En el caso de estos pronombres, efectivamente, los números parecen extremadamente discrepantes. En español se usa quince veces más este, en sus diferentes formas, que en portugués. O sea, comparado con el español, en portugués no se usa 
este. Este sería un primer elemento que se tendría que enseñar a los alumnos: que en español la distinción entre este y ese es significativa, y que este sí tiene que usarse. Solo así se puede explicar la variación relativamente pequeña que existe entre el uso de ese en ambas lenguas, y la gran diferencia que existe en el caso de este. En otras palabras este (castellano) y este (portugués) son falsos amigos de un tipo particular: significan lo mismo en las dos lenguas, pero se usan con una frecuencia distinta. Como siempre, aquí también la frecuencia tiene que ver con el registro. Si uno quisiera aventurar una hipótesis sería que en portugués el uso de este, opuesto a esse, indica un registro marcadamente elevado, mientras que la oposición este/ese en castellano indica una oposición espacial, sea esta literal o metafórica.

A título de curiosidad siguen los números del corpus Varsul que muestran, como se podía suponer, que en el lenguaje hablado el uso de este es todavía más restringido.

\begin{tabular}{|l|l|l|}
\hline & \multicolumn{1}{|c|}{ Varsul } & Portugués escrito \\
\hline Este & $1 / 8159$ & $1 / 4963$ \\
\hline Estes/estos & $1 / 266947$ & $1 / 27690$ \\
\hline Esta & $1 / 17915$ & $1 / 6862$ \\
\hline estas & $1 / 177964$ & $1 / 42224$ \\
\hline Promedio & $1 / 117746.25$ & $1 / 20434$ \\
\hline & & 15 \\
\hline Ese/esse & $1 / 716$ & $1 / 3164$ \\
\hline Esos/esses & $1 / 2215$ & $1 / 6631$ \\
\hline Esa/essa & $1 / 860$ & $1 / 3395$ \\
\hline Esas/essas & $1 / 1714$ & $1 / 7178$ \\
\hline Promedio & $1 / 1367.25$ & $1 / 5092$ \\
\hline & & 1.59 \\
\hline Aquel/aquele & $1 / 1281$ & $1 / 14177$ \\
\hline Aquellos/aqueles & $1 / 4809$ & $1 / 17960$ \\
\hline Aquella/aquela & $1 / 1313$ & $1 / 19203$ \\
\hline Aquellas/aquelas & $1 / 4818$ & $1 / 53110$ \\
\hline Promedio & $1 / 3055.25$ & $1 / 26112$ \\
\hline
\end{tabular}

Fig. 2. Demostrativos en el corpus Varsul y la Folha de

São Paulo 
La pregunta siguiente que podríamos hacernos es ¿cómo se hace entonces en portugués para distinguir entre este y ese, como se expresa en español? Creo que di con la respuesta cuando me riñó un día una colaboradora paraguaya al usar la secuencia "ese aquí" en español. Algunas búsquedas en Internet y en el corpus mostraron que tenía ella toda la razón. No encontré ningún ejemplo en ningún corpus de "este aquí, esta aquí" o "ese aquí, esa aquí". Esto me llevó a redactar el siguiente cuadro que refleja, me parece, de qué manera se tendría que enseñar los demostrativos en castellano para no cometer el error de enseñarles falsos amigos a los estudiantes.

\begin{tabular}{|l|l|}
\hline Español & Portugués \\
\hline Este & esse aqui \\
\hline Ese & Esse \\
\hline aquel & Aquele \\
\hline
\end{tabular}

FIG. 3. Traducción de demostrativos

La razón estructural es evidente. Con la desaparición de la distinción entre este y esse en portugués, se introdujo una nueva distinción por medio de una partícula auxiliar. Este recurso, sin embargo, no existe en castellano, ni hace falta que exista, ya que la distinción entre este y ese no se perdió.

\section{LÁSTIMA}

Lástima es un caso de falsos amigos de un tipo diferente. Cuando comparamos las definiciones de la palabra en los grandes diccionarios de español y de portugués, nos damos cuenta de que las acepciones coinciden, menos una. Me refiero a un uso que Houaiss define como "pessoa ou objeto sem préstimo - Ex.: teve um professor que era uma lástima", y Aurélio describe como "Depreciativo. Coisa ou pessoa inútil". De ahí que se puede escuchar a aprendices brasileños diciendo cosas como "mi auto es una lástima" o incluso "ese profesor es una lástima". Es uno de los muchos "falsos amigos" que no he encontrado en ninguna lista porque es de un tipo que no se considera tradicionalmente tal.

A título de ilustración busqué algunos ejemplos en Internet de lástima en portugués. Me llamó la atención que el sentido despreciativo, que solo existe en portugués, fuera más frecuente que el sentido 'compasivo', que es el único en español. A seguir cito algunos ejemplos de frases, clasificándolas según puedan usarse de manera idéntica en castellano y portugués o no. 


\begin{tabular}{|c|c|}
\hline Mismo sentido que en castellano & Otro sentido \\
\hline É uma lástima que seja assim & Fazer entrevista é uma lástima \\
\hline $\begin{array}{l}\text { Às vezes fico pensando que é uma lástima a } \\
\text { vida ser tão curta }\end{array}$ & A televisão brasileira é uma lástima \\
\hline $\begin{array}{l}\text { É uma lástima que o país ceda às pressões de } \\
\text { grupos religiosos }\end{array}$ & $\begin{array}{l}\text { O vestibular como ordenador do sistema de } \\
\text { ensino é uma lástima }\end{array}$ \\
\hline \multirow{3}{*}{$\begin{array}{l}\text { É uma lástima que muitos avós jovens não } \\
\text { busquem orientação ou assessoramento }\end{array}$} & Massa sem molho é uma lástima \\
\hline & A geração Ananias e Safira é uma lástima \\
\hline & $\begin{array}{l}\text { O coringão é uma lástima em Copas Liberta- } \\
\text { doras }\end{array}$ \\
\hline
\end{tabular}

Fig. 4. Traducción de lástima

En el caso de las frases de la columna a la izquierda, lástima puede traducirse en castellano por la misma palabra (lástima). En la columna de la derecha las traducciones serían varias. Podría ser 'pérdida de tiempo', 'desgracia', 'tristeza', 'horror'. Como se puede notar, hay aquí un buen ejemplo de cómo la gramática tiene su significación semántica ${ }^{7}$. En todas las frases en las que lástima significa pena, la secuencia es Verbo-Atributo-Sujeto, mientras cuando significa horror, o algo semejante, la secuencia es Sujeto-Verbo-Atributo ${ }^{8}$.

\section{HABLAR/ FALAR; DECIR/DIZER}

Llegamos ahora al cuarto tipo de falsos cognados que es el de palabras que se oponen en cada una de las lenguas, con acepciones aparentemente idénticas, pero que obedecen a restricciones gramaticales, léxicas, o ambas, diferentes. Tanto en portugués como en español, hablar y decir, por un lado, y falar y dizer, por otro, se reparten el campo de la expresión verbal de una manera aparentemente comparable 9 . En castellano hablar es, según el DRAE: "Articular, proferir palabras para darse a entender" y decir "Manifestar con palabras el pensamiento".

7 Es el caballo de batalla de los funcionalistas británicos (Halliday 1994 y Sinclair 1991).

8 Agradezco a Pablo Cardellino esta observación.

9 La cuestión fue extensamente tratada en Blaszkowski de Jacobi (2001). 
En portugués, según el Aurélio, falar es "dizer palavras; expressar-se ou exprimir-se por meio de palavras; dizer" y dizer es "exprimir por palavras; enunciar". La diferencia entre dizer y falar es, como se sabe, sobre todo una cuestión de registro. Dependiendo del contexto, dizer es más formal que falar.

Sin embargo, por las semejanzas de sentido, la tentación de usar dizer cuando se usa decir, y falar cuando se usa hablar, es muy grande. Sabemos que no es correcto, pero como en la práctica este uso no compromete la comprensión, la tendencia es de mezclar los dos pares de verbos.

El DRAE solo menciona dos casos en que hablar puede usarse de manera transitiva. El primero es "17. Emplear uno u otro idioma para darse a entender. HABLA francés; HABLA italiano y alemán". El otro es "18. Decir algunas cosas especialmente buenas o malas. HABLAR pestes; HABLAR maravillas". Creo que podemos añadir a este uso otro que se podría explicar como siendo una forma de conversar. En castellano se puede también "hablar política"".

Ocurre que viviendo como hispanohablante, en Brasil cualquiera empieza a sentir la influencia de falar que, en portugués, es casi un sinónimo de dizer. Oficialmente estos verbos no son falsos amigos. Sí lo son en el sentido en que no comprometen la comprensión, pero sí la naturalidad. "Falei pra ele" es "le dije" o "se lo dije"; "falei tudo que queria" es "(le) dije todo lo que quería".

Aunque los números no lo dicen todo, es interesante comparar la frecuencia de las parejas falar/dizer, hablar/decir en el corpus.

\begin{tabular}{|l|l|}
\hline Falar & $1 / 7443$ \\
\hline Dizer & $1 / 6512$ \\
\hline & \\
\hline Hablar & $1 / 10148$ \\
\hline Decir & $1 / 2072$ \\
\hline
\end{tabular}

FIG. 5. Frecuencia de falar/dizer, hablar/decir

Aquí también lo que primero llama la atención es la disparidad de las frecuencias. En portugués falar y dizer parecen usarse casi con la misma frecuencia, mientras que en castellano se usa mucho más decir que hablar. Este hecho corrobora nuestra hipótesis según la cual se usa en portugués muchas veces falar cuando en castellano solamente se usa decir. Falsos cognados de este tipo son, sin lugar a duda, mucho más difíciles de detectar, y mucho más difícil es enseñar el uso correcto.

10 Un estudio más pormenorizado, en preparación, muestra un cuadro más complejo aún, con diferencias regionales como en el caso de Chile. 


\section{CONCLUSIÓN}

La mayoría de los estudiantes brasileños quiere aprender a expresarse no solamente de una manera gramaticalmente correcta, sino también de una manera natural. Ocurre que cuando un brasileño le dice a un hispanohablante que "está sentado en el balcón”, queriendo decir "que está sentado en el mostrador", habrá un error de comprensión que la realidad se encargará de corregir muy rápidamente. Ahora, si este mismo brasileño le dice al hispanohablante que "está todo esclarecido"11, no habrá ningún problema de comprensión, habrá un error de naturalidad. Este tipo de problema es mucho más frecuente de lo que generalmente se admite y no se le dedica ninguna atención en los diccionarios tradicionales.

En este artículo he querido mostrar que el problema de los "falsos amigos" en portugués y español es más complejo de lo que parece. Cuando tradicionalmente se limita el problema a pares de palabras que son iguales pero significan otra cosa, espero haber demostrado que los verdaderos problemas causados por la proximidad de las dos lenguas están en diferencias de registro, de coincidencia parcial de los campos semánticos o de usos gramaticales diferentes. Si dispusiéramos de un "diccionario de falsos amigos" que incluyera estos casos de descripción más compleja, tendríamos una léxico-gramática contrastiva del portugués y del castellano que sería de una tremenda ayuda en la enseñanza. Hay una infinidad de temas que esperan ser investigados. Pienso en los pares buscar/procurar; colocar/poner; percibir/perceber; similar/semejante/semelhante; algo/alguma coisa; assumir/asumir; ganhar/ganar; porém/pero, y otros muchos. Ninguno de esos "falsos amigos" se encuentra hoy en los diccionarios de falsos amigos disponibles en el mercado. Mientras sigamos pensando que el problema de los falsos amigos es el de confusión de sentidos pura y simplemente, seguiremos limitándonos a la solución de un problema fácil, y que resuelve muy poco.

\section{REFERENCIAS BIBLIOGRÁFICAS}

Actas del Congreso Brasileño de Profesores de Español (1996): "Dificultades para la enseñanza del español a lusohablantes", Brasília, Associação de Professores de Espanhol do Distrito Federal-Consejería de Educación de la Embajada de España.

Alcina Franch, J. y J. M. Blecua (1975): Gramática española, Barcelona, Ariel.

Alves SoARes, J. (2002): “Los heterosemánticos en español y portugués. Un desafío a la lectura/interpretación: el caso de los ‘vestibulandos' brasileños”, en Procee-

11 "Está todo esclarecido": ninguna ocurrencia en Google. "Está tudo esclarecido": 373 ocurrencias en Google. 
dings of the 2. Congreso Brasileño de Hispanistas, São Paulo (SPSPSP, Brasil) [online, citado 29 Octubre 2004; <http://www.proceedings.scielo.br/ scielo.php?script=sci_arttext\&pid=MSC0000000012002000100032\&lng=en\& $\mathrm{nrm}=\mathrm{iso}>$ ].

BlaszKowsKi DE JACOBI, C. C. (2001): Lingüística de Corpus e Ensino de Espanhol a Brasileiros: Descrição de padrões e preparação de material didático (decir) hablar; mismo; mientras/en cuanto aunque, São Paulo, Pontífica Universidade Católica de São Paulo, Tesis de maestría inédita.

Cunha, C. y L. F. L. CintRA (1985): Nova gramática do português contemporâneo, Rio de Janeiro, Nova Fronteira.

DRAE. Real ACAdEMia Española (200122): Diccionario de la lengua española, Madrid, Espasa-Calpe.

FeIJóo Hoyos, B. L. (1992): Diccionario de falsos amigos del español y del portugués / Dicionário de falsos amigos do espanhol e do português, Brasília, Embajada de España-Editora Página Aberta.

Halliday, M. A. K. (1994²): Introduction to Functional Grammar, London, Edward Arnold.

Marzano Mendes, F. (2001): Dicionário Espanhol-Português de falsas semelhanças, Rio de Janeiro, Campus.

Mello T. de y S. BATH (1996): Amigos traiçoeiros, Brasília, Universidade de Brasília.

ReAl ACADEMia Española (1973): Esbozo de una nueva gramática de la lengua española, Madrid, Espasa-Calpe.

Sinclair, J. (1991): Corpus, Concordance, Collocation, Oxford, Oxford University Press.

SABINo, M. A. (2002): Falsos cognatos, falsos amigos ou cognatos enganosos? Desfazendo a confusão teórica na prática, São Paulo, UNESP, inédito. 